\title{
Cellular Magnetic Resonance Imaging
}

3

12 Address correspondence to:

13 Natasha N. Knier

14 Robarts Research Institute,

15 Schulich School of Medicine and Dentistry,

16 Western University

17 London, Ontario, N6A 5B7,

18 Canada

19 Email: nknier@uwo.ca

20

21

22 Keywords: breast cancer, brain metastasis, cellular magnetic resonance imaging, iron oxide nanoparticles, mouse 23 models

\author{
Natasha N. Knier ${ }^{1,2}$, Amanda M. Hamilton ${ }^{1}$, and Paula J. Foster ${ }^{1,2}$
}

\author{
${ }^{1}$ Imaging Research Laboratories, Robarts Research Institute, London, ON, Canada \\ ${ }^{2}$ Department of Medical Biophysics, Western University, London, ON, Canada
}




\section{Abstract}

26 Metastasis is the leading cause of mortality in breast cancer patients, with brain metastases becoming increasingly

27 prevalent. Studying this disease is challenging due to the limited experimental models and methods available. Here,

28 we used iron-based cellular MRI to track the fate of a mammary carcinoma cell line (MDA-MB-231-BR) in vivo to

29 characterize the growth of brain metastases in the nude and severely immune-compromised NOD/SCID/ILIIrg-/-

30 (NSG) mouse.

31 Nude and NSG mice received injections of iron-labeled MDA-MB-231-BR cells. Images were acquired with a 3T

32 MR system and assessed for signal voids and metastases. The percentage of signal voids and the number and volume

33 of metastases were quantified. Ex vivo imaging of the liver, histology, and immunofluorescence labeling was

34 performed.

35 On day 0 , iron-labeled cells were visualized as signal voids throughout the brain. The percentage of voids decreased

36 significantly between day 0 and endpoint. At endpoint, there was no difference in the number of brain metastases or

37 tumour burden in NSG mice compared to nudes. Tumour volumes in nude mice were significantly larger than in

38 NSG mice. Body images indicated that the NSG mice had metastases in the liver, lungs, and lymph nodes.

39 Characterization of the NSG and nude mouse is necessary to study breast cancer brain metastasis in vivo. Here, we

40 show that the 231BR cell line grew differently in NSG mice compared to nude mice. This work demonstrates the

41 role that imaging can play toward credentialing these models that cannot be done with other in vitro or 42 histopathologic methods alone. 


\section{Background}

45 Breast cancer is a leading cause of death in women mainly due to the propensity of breast tumors to metastasize to

46 regional and distant sites, such as the lymph node, lung, liver, bone and brain [1]. The incidence of brain metastases

47 is increasing due to the introduction of more sensitive diagnostic methods and improved systemic therapies leading

48 to improvements in extra-cranial control and survival [2-4]. Breast cancer is a disease with a number of subtypes and

49 patients with metastatic 'triple-negative' breast cancer tend to develop brain metastasis at a high rate [5, 6]. For the

50 HER2 amplified subtype, the frequency of brain metastasis has been reported to be as high as 50\% [7].

52 Once a metastatic cancer cell arrives in the brain one of three things can happen: (1) it may die, (2) it may proliferate 53 to form micrometastases, or (3) it may remain viable but dormant ('non-proliferative') [8, 9] If the solitary cells 54 proliferate to form micrometastases, they may again experience one of three fates: (1) they may die, (2) they may 55 continue to proliferate and form macrometastases, or (3) they may persist as "dormant" micrometastases, where 56 dormancy is defined as a balance between proliferation and apoptosis within the cell population such that there is no 57 net growth $[9,10]$. The factors that tip the balance between dormancy and proliferation are poorly understood. Both 58 dormant single cells and dormant micrometastases are believed to be sources of cells that contribute to tumor 59 recurrence [11]. Dormant cancer cells also present a substantial therapeutic problem; since they are quiescent, they 60 are non-responsive to current therapies which target proliferating cells [8].

62 Currently, only a handful of models specific to breast cancer brain metastasis have been described and even fewer 63 allow for in vivo investigation of cancer cell dormancy. Both murine and human cancer cell lines have been 64 developed to mimic as many steps in the metastatic cascade as possible. A well characterized murine breast cancer 65 cell line is the 4T1-BR5 cell line, a highly tumorigenic and invasive cell line that has undergone multiple rounds of 66 selection to preferentially grow in the mouse brain [12]. There are several advantages to using a murine breast 67 cancer cell line to study brain metastasis, as growth and maintenance is easy and relatively inexpensive, and it can 68 be grown in immune-competent mice, which is of particular interest for studying this disease in a way that 69 recapitulates the tumor microenvironment [13]. 
71 A small number of human breast cancer brain metastatic cell lines have also been developed, including the 72 commonly used MDA-MB-231BR (231BR), MDA-MB-231BR-HER2, MA11, JIMT1-BR3, SUM190-BR3 cell

73 lines. The MDA-MB-231BR cell line has been particularly well characterized for studying the progression of brain

74 metastases in nude mice. Nude mice are the most commonly used immune-deficient strain for models which use 75 human cell lines. They have a genetic mutation that causes a deteriorated or absent thymus, resulting in a lack of T 76 cells [14]. The 231BR cell line grows selectively in the brain of nude mice, without metastatic growth seen in other 77 distant organs [15]. Human breast cancer cell lines are one of the most widely used models to study the metastatic growth of cancer in vitro and in vivo, as they have been used to provide extensive insight into the characteristics of

79 human cells and can be used for high throughput screening of various drugs [16]. There are, however, significant

80 limitations to using human and murine breast cancer cell lines, as the quick progression in vivo can limit 81 opportunities for adequate therapeutic testing, and the growth of these cells in vitro prior to establishment in a mouse 82 can cause changes in the genetic composition due to clonal selection [17]. Many groups have shown that these 83 genetic changes result in these models failing to maintain tumor heterogeneity, which is now recognized as a critical 84 element for developing personalized treatments [18-20]. Studying breast cancer with immunocompetent mice also 85 has limitations, as tumor latency and growth can be variable and slow [21]. There is also increased difficulty in 86 establishing human tumors successfully in these mice. Immune-compromised mice lack a comparable tumor

87 microenvironment to clinical tumors and fail to provide a realistic result of interactions with the natural immune 88 response, particularly in the case of studying anticancer therapeutics [22].

90 In more recent years, researchers have moved towards studying breast cancer and its subtypes with patient-derived 91 xenografts (PDX), which allow for the growth of human primary breast cancer tissue that has been recently resected 92 from consenting patients into immune-compromised rodents [23]. NOD/SCID/ILIIrg ${ }^{-/-}$(NSG) mice are the preferred 93 strain of mice to engraft a PDX, as they are highly permissive to growing breast cancer metastasis, and resembles 94 the metastatic pattern seen in human patients. NSG mice are the most immunodeficient mouse strains to date, 95 lacking T cells, B cells, NK cells, and have defective macrophages and dendritic cells [24-26]. Even fewer PDX 96 models have been developed specific to breast cancer brain metastasis, including the novel F2-7 and E22-1 PDX cell 97 lines [27], as well as others who have established low passage PDX models of breast cancer brain metastasis and 98 those that implant fresh tumor tissue directly into the rodent brain [28]. 
99 The D2.0R and related D2A1 mouse mammary tumor cells have been well characterized as model systems for

100 tumor cell dormancy. In mice, D2.0R/R cells invade distant metastatic sites, remain as single quiescent cells for

101 prolonged periods of time, and occasionally proliferate into metastatic tumors. D2A1/R cells, have a much shorter

102 dormancy period before forming metastases in the lung, liver, and other organs [29]. A human breast cancer cell

103 line, MCF-7 has also been studied to examine the mechanisms of dormancy, however it is poorly metastatic [30].

104 For all of these brain metastasis models - cancer cell growth, metastasis and dormancy are typically studied using 105 methods such as histology, flow cytometry, immunohistochemistry, and fluorescent microscopy [31]. While these

106 techniques provide useful information about molecular and cellular markers and morphology, they are limited to

107 studying this disease after endpoint has been reached. There is a clear need to characterize brain metastatic models

108 of breast cancer in vivo, which can be accomplished through use of imaging modalities and cell tracking techniques.

110 Cellular magnetic resonance imaging (MRI) combines the ability to obtain high resolution MRI data with the use of 111 iron oxide nanoparticles for labeling specific cells, thereby enhancing their detectability [32, 33]. The presence of 112 the iron in cells causes a distortion in the magnetic field and leads to abnormal signal hypo-intensities in iron113 sensitive images (T2*-weighted images are most often used). Areas containing iron labeled cells therefore appear as 114 regions of low signal intensity on MRI images, creating negative contrast [34]. We have previously shown that it is 115 possible to use cellular MRI to track iron-labeled 231BR cancer cells in the nude mouse brain [35-37]. Proliferative 116 cancer cells lose the iron label over time; as the cells divide the particles are apportioned to daughter cells and

117 eventually some cells contain too little iron to be detectable by MRI. As brain metastases form, changes to the tissue 118 result in the tumor appearing brighter than the surrounding brain in MRI. Nonproliferative cancer cells retain the 119 iron particles and can be detected over long periods of time [37].

121 In this study, we used these cellular MRI techniques to characterize and compare the growth of 231BR brain 122 metastases and the persistence of iron-retaining cancer cells in nude and NSG mice. Our goal was to evaluate the 123 NSG mouse as a model for breast cancer brain metastasis and dormancy.

\section{Methods}

\section{Cell Culture and MPIO Labeling Procedure}


127 Brain trophic human breast cancer cells (MDA-MB-231BR) expressing green fluorescent protein (GFP) were

128 maintained with Dulbecco's modified Eagle's medium containing 10\% fetal bovine serum (complete DMEM) at

$12937^{\circ} \mathrm{C}$ and $5 \% \mathrm{CO}_{2} .2 \times 10^{6}$ of these cells were seeded and allowed to adhere for 24 hours. To iron label these cells,

130 cells were supplemented with $25 \mu \mathrm{g} \mathrm{Fe} / \mathrm{mL}$ of micron-sized iron oxide particles (MPIO) (0.9 $\mu \mathrm{m}$ diameter, 63\%

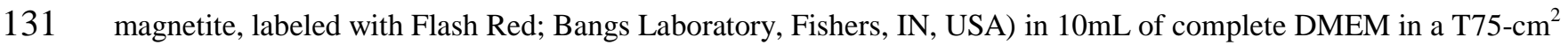

132 flask. Following this, cells were washed once with phosphate buffered solution (PBS) within the flask and

133 trypsinized with $0.25 \%$ Trypsin-EDTA. After, cells were harvested and washed three additional times with PBS in

134 the flask to thoroughly remove unincorporated MPIO prior to cell injections. Cell viability was assessed and

135 calculated using the Trypan blue exclusion assay. To visualize MPIO labelling, labeled cells were affixed to a glass

136 slide with a ThermoFisher Cytospin 4 cytocentrifuge and fixed with a Methanol/Acetic acid solution. Slides were

137 then stained with a Perl's Prussian Blue (PPB) solution and counterstained with Nuclear Fast Red. Slides were

138 dehydrated with increasing concentrations of ethanol, cleared with xylene, and coverslipped with a xylene-based

139 mounting medium. These PPB-stained slides were examined to assess the localization of MPIO within the cell and

140 to determine the labeling efficiency using a Zeiss AXIO Imager A1 Microscope (Zeiss Canada, Toronto, ON,

141 Canada). Iron oxide nanoparticles appear dark blue, and cells appear light pink in colour.

143 Animal Model and Work Flow

144 All animals were cared for in accordance with the standards of the Canadian Council on Animal Care, under an 145 approved protocol of the University of Western Ontario's Council on Animal Care and housed in a pathogen-free 146 barrier facility. Female nude mice (nu/nu Foxn1, aged 6-8 weeks, from Charles River Laboratories, Wilmington, 147 MA) and female NSG mice (NOD.Cg-Prkdc $c^{\text {scid }} I l 2 r g^{\text {tml } w j l} /$ SzJ, 6-8 weeks, Humanized Mouse and 148 Xenotransplantation Facility, Robarts Research Institute, University of Western Ontario, London, ON) were first 149 anesthetized with isoflurane ( $2 \%$ in $100 \%$ oxygen). Nude $(n=10)$ and NSG mice $(n=10)$ were then injected with a 150 suspension of $1.5 \times 10^{5}$ MPIO-labeled MDA-MB-231BR/GFP+ cells in $100 \mu \mathrm{L}$ of sterile saline and $15 \%$ Vevo 151 MicroMarker microbubble solution (FUJIFILM, VisualSonics Inc., Toronto, ON, Canada). Cell suspension was 152 loaded into a $100 \mu \mathrm{L}$ Hamilton syringe with a $30 \mathrm{G}$ needle. Cells were administered by slow intracardiac injection 153 into the beating left ventricle of the heart with ultrasound imaging guidance on a Vevo 2100 ultrasound system

154 (FUJIFILM, VisualSonics Inc., Toronto, ON, Canada). Cancer cell arrest was evaluated with MRI on the day of the 
155 injection (Day 0) and used to determine whether mice had a successful intracardiac injection. Absence of signal

156 voids in the brain indicated an unsuccessful injection, resulting in exclusion from the remainder of the study. Nude

157 mice with successful injections had MRI performed at day 21 and day 32 post-injection. NSG mice with successful

158 injections had MRI performed at day 21 post-injection (Figure 1).

159 Additionally, 1 nude mouse and 3 NSG mice received intracardiac injections with $1.5 \times 10^{5}$ MPIO labeled MD-MB-

$160231 \mathrm{BR} / \mathrm{GFP}+$ cells and intraperitoneal injections of $200 \mu \mathrm{L}$ of gadopentetate dimeglumine (Magnevist, Schering,

161 US; $0.5 \mathrm{mmol} / \mathrm{mL}$ ) on Day 0 to assess cancer cell arrest in the liver. These mice were sacrificed on Day 0 by

162 isoflurane overdose approximately 40 minutes after intraperitoneal administration. These mice were then

163 exsanguinated, and brains and livers were removed for ex vivo imaging.

\section{Magnetic Resonance Imaging}

166 All brain and body MRI examinations were acquired on a 3.0 Tesla GE MR750 clinical MR scanner (General 167 Electric, Mississauga, ON, Canada) using a custom-built gradient coil (inner diameter $=17.5 \mathrm{~cm}$, gradient strength $=$ $168500 \mathrm{mT} / \mathrm{m}$, and peak slew rate $=3000 \mathrm{~T} / \mathrm{m} / \mathrm{s}])$. Brain and ex vivo liver images used a custom-built solenoidal mouse 169 brain radiofrequency (RF) coil. Body images were acquired using a $4.3 \times 4.3 \mathrm{~cm}$ dual tuned ${ }^{1} \mathrm{H} /{ }^{19} \mathrm{~F}$ surface coil

170 (Clinical MR Solutions, WI, USA), originally built for imaging small ROIs in humans. Mice were anesthetized with $1712 \%$ isoflurane in $100 \%$ oxygen administered through a nose cone. In vivo brain and body images were acquired 172 using a 3D balanced steady-state free precession (bSSFP) sequence [Fast Imaging Employing Steady State 173 Acquisition (FIESTA) on a GE system] that has been optimized for simultaneous detection of signal voids produced 174 by iron-loaded cells and hyperintense metastases. This permitted the assessment of both cell arrest and retention as 175 well as the number and volume of metastases throughout the mouse brain. Brain images for nude mice were 176 acquired on day 0 , day 21 , and day 32. Brain imaging for NSG mice occurred on day 0 and day 21. Scanning 177 parameters were as follows: resolution $=100 \times 100 \times 200 \mu \mathrm{m}$, repetition time $(\mathrm{TR})=7 \mathrm{~ms}$, echo time $(\mathrm{TE})=3.5 \mathrm{~ms}$, 178 bandwidth $(\mathrm{BW})=20.83 \mathrm{kHz}$, flip angle $(\mathrm{FA})=35$ degrees, signal averages $=2$, phase cycles $=8$, matrix $=150 \mathrm{x}$ 179150 , scan time = approximately 33 minutes per mouse. Body images were acquired on Day 20. Body imaging 180 parameters were as follows: resolution $=200 \times 200 \times 200 \mu \mathrm{m}$, repetition time $(\mathrm{TR})=4.7 \mathrm{~ms}$, echo time $(\mathrm{TE})=2.3$, 181 bandwidth $(\mathrm{BW})=+/-31.25 \mathrm{kHZ}$, flip angle $(\mathrm{FA})=35$ degrees, signal averages $=2$, phase cycles $=8$, matrix $=250$ 182 x 250, scan time = approximately 20 minutes. Ex vivo liver imaging parameters were acquired using a spoiled 
183 gradient echo sequence that also allows for the detection of signal voids throughout the liver. Ex vivo liver samples

184 were immersed in tubes containing Fluorinert ${ }^{\mathrm{TM}}$ FC-40 Electronic Liquid (3M, St. Paul's, MN, USA), a

185 fluorocarbon liquid which produces a black background in proton images. Images were taken of ex vivo livers

186 acquired on day 0 for both strains, day 21 for the NSG mouse, and day 32 for the nude mouse. The scanning

187 parameters for ex vivo liver images were as follows: resolution $=100 \times 100 \times 200 \mathrm{um}$, repetition time $(\mathrm{TR})=43 \mathrm{~ms}$,

188 echo time (TE) $4.844 \mathrm{~ms}$, bandwidth $(\mathrm{BW})=31.25 \mathrm{kHz}$, flip angle $(\mathrm{FA})=60$ degrees, signal averages $=1$, phase

189 cycles $=8$, matrix $=250 \times 250$, scan time $=$ approximately 23 minutes.

\section{Image Analysis}

192 Images were analyzed using open-source OsiriX image software (Pixmeo, SARL, Bernex, Switzerland), version

193 10.0.4 and Horos image software, version 3.3.5. Brain images were evaluated for successful cell delivery by

194 assessment of signal voids on day 0. To quantify cancer cell arrest throughout the brain, the number of black pixels

195 within the total brain volume was determined from day 0 MRI images. The brain was manually segmented as a

196 region of interest and then a threshold value was determined based on the mean pixel intensity value of a

197 representative signal void within the brain region \pm 2 standard deviations. The total number of black pixels under

198 this threshold value within the entire brain volume signal was obtained from a pixel intensity histogram. To quantify

199 brain metastases at day 21 and day 32, tumors were counted in all image slices by a single observer. Each tumor was

200 manually segmented, and the tumor volumes were reconstructed using the OsiriX and Horos volume algorithm. To

201 calculate brain tumor burden at day 21 and day 32, an ROI was drawn around the outline of the brain in each slice,

202 and a 3D reconstruction using the OsiriX and Horos volume algorithm provided a quantification of the total brain

203 volume. The total volume of all segmented tumors was then determined by adding each individual tumor volume

204 measurement and calculating the percentage of the total brain volume occupied by tumors. Body images were

205 qualitatively assessed for the presence of metastases in the liver, lung, and lymph nodes. All quantitative values

206 were presented as the mean \pm standard error. Statistical analysis was performed using Welch's $t$ tests on GraphPad

207 Prism version 8 software (GraphPad, San Diego, CA). 
210 At each strain's respective endpoints, mice were euthanized by isofluorane overdose and perfused with $4 \%$

211 paraformaldehyde. Brains were removed and placed into paraformaldehyde for another 24 hours. Fixed brains were

212 processed, paraffin embedded and then cut into 6 or 8 um sections. Sliced sections were deparaffinized and stained

213 with either hematoxylin and eosin (H\&E) or immunofluorescently labelled for Ki67.

\section{Hematoxylin and eosin (H\&E) staining}

216 Sections were washed briefly in distilled water, stained in Harris hematoxylin solution for 5 minutes and 217 differentiated in $1 \%$ acid alcohol for 30 seconds. After washing in $0.2 \%$ ammonia for 5 minutes, sections were 218 counterstained in eosin-phloxine solution for 30 seconds; dehydrated through $95 \%$ alcohol, 2 changes of absolute 219 alcohol, and 5 minutes each. Sections were then dehydrated and cleared through 95\% ethyl alcohol, absolute alcohol 220 and xylene, and mounted with resinous medium.

\section{Immunofluorescent labelling}

223 Ki-67 immunostaining was performed using a rat anti-Ki-67 antibody (1:400 dilution; Catalog \#14-5698-82,

224 Invitrogen). All sections were permeabilized with $0.2 \%$ Triton X-100 in PBS for 15 minutes and non-specific 225 protein binding was then blocked by incubation in a commercial blocking agent (ab156024, Abcam) for 1 hour at 226 room temperature. Sections were then incubated with the Ki-67 primary antibody in commercial antibody dilutant

227 (ab64211, Abcam) at room temperature for 1 hour. Negative controls (without addition of primary antibody) were 228 performed on adjacent sections. Unbound primary antibody was washed away through three 5-minute exchanges of 229 1xPBS. Sections were then incubated with an anti-rat Alexa Fluor-488 secondary antibody (1:300 dilution; Catalog 230 \#A-11006, Invitrogen) for 1 hour. Unbound secondary antibody was washed away through three 5-minute 231 exchanges of 1xPBS. Finally, nuclei were counterstained with Hoechst 33258 for 5 minutes and rinsed sections 232 cover slipped for microscopic examination.

\section{Results}

235 In Vitro Studies

$236 \quad$ Cell Labelling 
237 MDA-MB-231BR cells were efficiently labeled with MPIO, as demonstrated by the Perl's Prussian blue staining of 238 cells shown in Figure 2a; cancer cells appear pink, and the intracellular iron is blue. Labelling efficiency of $23996.33 \pm 1.20 \%$ was achieved with $97.33 \pm 0.88 \%$ cell viability.

\section{$241 \quad$ In Vivo Studies}

\section{Experimental Endpoint}

243 In our experience, intracardiac injection of $1.5 \times 10^{5}$ 231BR cells in nude mice results in brain tumor burden, 244 significant weight loss and neurological impairments which leads to a requirement for euthanasia at 28-34 days post 245 injection. In this study the nude mice reached experimental endpoint on day 32. In contrast, NSG mice could only 246 be studied until day 21 , at which time they had reached a weight loss greater than $15 \%$ of their body weight, 247 resulting in extreme cachexia and anorexia. Additionally, these mice showed severe signs of neurological 248 impairment, resulting in the inability to perform normal functions and exhibiting paralysis and circling. Post-mortem 249 examination also revealed significant liver tumor burden, which also contributed to the early, unexpected endpoint.

\section{$251 \quad$ Imaging Cell Arrest and Retention}

252 On day 0, bSSFP brain images confirmed the successful intracardiac injection of MPIO-labeled cancer cells in all 253 mice; iron-labeled cancer cells appeared as distinct regions of signal void throughout the brain due to their initial 254 arrest in brain vasculature (Figure 2b). Figure 3a shows representative day 0 images for each mouse strain. When 255 quantifying the percentage of the brain consisting of black signal voids, approximately $4.69 \% \pm 1.71$ and $6.26 \% \pm 1.86$ 256 of the brains of NSG and nude mice contained arrested cancer cells, respectively. At each strain's endpoint, the 257 number of black pixels was again determined, with percentages significantly reduced to approximately $1.89 \% \pm 0.57$ 258 in NSG mice (day 21) and $2.45 \% \pm 0.48$ in nude mice (day 32). While the number of arrested cancer cells 259 significantly differs from day 0 to endpoint for both NSG $(p=0.03)$ and nude mice $(p=0.02)$, there was no 260 significant differences between strains at each time point (Figure 3b).

\section{Day 21 MRI}

263 On day 21, metastases were detectable in bSSFP brain images as regions of signal hyperintensity in both strains of 264 mice (Figure 4a). On day 21, the mean number of MRI-detectable metastases was significantly higher in NSG mice 
(63.70 \pm 5.37$)$ compared to nude mice (15.33 \pm 4.29$)$ (Figure $4 \mathrm{~b}$ ). While the nude mice had fewer tumors, they were significantly larger. The mean volume of tumors in nude mice was $0.06 \mathrm{~mm}^{3} \pm 0.01$, compared to $0.04 \mathrm{~mm}^{3} \pm 0.003$ for NSG mice (Figure 4c). This mean volume was based on a total number of 637 tumors in all NSG mice and 72 tumors counted in all nude mice. At day 21, the mean tumor burden was significantly higher for NSG mice (2.39 $\mathrm{mm}^{3} \pm 0.18$ ) compared to nude mice $\left(0.82 \mathrm{~mm}^{3} \pm 0.35\right)$ (Figure 4D).

\section{Day 32 MRI}

272 Nude mice were followed out their endpoint of day 32 and imaging analysis was compared to the endpoint data for

273 the NSG mice (Figure 5). The mean number and volume of brain metastases in nude mice increased between days

27421 and 32. On day 32 the mean number of brain metastases in nude mice was $39.60 \pm 10.99$. When comparing the 275 mean number of metastases for NSG and nude mice at their respective endpoints there was no longer a significant 276 difference $(\mathrm{p}=0.07)$ (Figure 5b).

277 On day 32 the mean volume of brain metastases in nude mice was $0.32 \pm 0.23 \mathrm{~mm}^{3}$, compared to $0.04 \pm 0.03 \mathrm{~mm}^{3}$ on 278 day 21 . This was significantly higher $(\mathrm{p}=<0.0001)$ than the mean volume of brain metastases at the NSG endpoint 279 (Figure 5c). Accordingly, nude mice had significantly more tumor burden than NSG mice (Figure 4d), which was a 280 reversal from the previous timepoint at day 21. Due to the earlier endpoint of NSG mice but lesser brain tumor 281 burden, body MRI was acquired for an NSG mouse at day 20. Notably, there was significant tumor burden detected 282 by MRI within the liver, lungs (Figure 6A), and lymph nodes (Figure 6b) of the NSG mice. Nude mice had no MRI283 detectable tumors within the body outside the brain (Figure 6c).

\section{Ex Vivo Liver MRI}

286 Discrete signal voids were visible in the day 0 images of $e x$ vivo livers from each strain of mouse, suggesting the 287 arrest of iron labeled 231BR cells throughout the liver (Figure 6d,e). Ex vivo liver mages obtained from mice at 288 endpoint showed numerous regions of abnormal high signal intensity associated with liver metastases, confirming 289 the in vivo image results (Figure 6f). A photo of a representative liver sample clearly shows the liver metastases on 290 the surface (Figure 6h). No regions of abnormal signal hyperintensity were observed in ex vivo images of the nude

291 mouse liver (6g). H\&E stained sections of the liver tissue from NSG mice confirmed the presence of metastases (6i) 292 and ki67 staining showed that they were highly proliferative (6j). 


\section{Discussion}

294 This work demonstrates for the first time the use of in vivo longitudinal MRI based cell tracking to compare murine

295 models of brain metastatic breast cancer. We show that there are significant differences in tumor progression for

296 231BR cells in NSG versus nude mice. While the initial arrest, clearance, and retention of iron-labeled cells was

297 similar, brain metastases developed more quickly in NSG mice and NSG mice developed substantial body tumor

298 burden, particularly in the liver, leading to an earlier endpoint.

The 231BR cell line was developed by Yoneda et al [15]. and is a brain-colonizing subline of the metastatic triple-

301 negative MDA-MB-231 human breast cancer cell line, which was isolated by six repeated cycles of intra-cardiac

302 injection and harvesting from brain metastases grown in nude mice. In our lab, the intra-cardiac injections are

303 performed using ultrasound (US) guidance. Of note, was our observation by US of a thicker heart muscle

304 surrounding the left ventricle of the NSG mouse. This anatomical observation made intracardiac injections more

305 difficult, as the ventricular space appeared smaller with US. The observation of signal voids in brain images

306 acquired on day 0 was used to identify successful delivery of cells to the brain. In this study, all mice imaged had

307 successful injections and were included in the study. Delivery of cells to a specific organ is related to the cardiac

308 output that is delivered to that organ. The cardiac output to the brain of a mouse is $~ 9.5 \%$ [38]. Here, we injected

309150,000 cells into the left ventricle of the heart, and therefore we can expect $\sim 14,250$ cells to be successfully

310 delivered to the brain. Only a percentage of these cells will arrest; previous studies, however, have shown that less

311 than $1 \%$ of cells are retained in the microcirculation of the brain after 2 hours post-injection [38]. In previous

312 studies, we have used PPB staining and fluorescence microscopy to demonstrate that these signal voids correspond

313 to the presence of iron-labeled cells in the brain $[35,36]$.

315 The size of the signal void created by iron-labeled cells is much larger than the actual cell size, due to what is known

316 as the blooming effect. The blooming effect is a susceptibility artifact that occurs as a result of the iron oxide

317 nanoparticle, which causes a local magnetic field inhomogeneity [34]. We have previously shown that we can detect

318 single iron-labeled cells arrested in the mouse brain using cellular MRI [35, 39, 40]. Our image showed that the

319 number of signal voids in the brain was similar for nude and NSG mice on day 0 and at endpoint. While we 320 acknowledge that there is potential for immune cell uptake of the iron oxide nanoparticles that may be released by 
321 dead cancer cells, we believe that the majority of the signal voids that remain present are live cancer cells retaining 322 iron. For example, in Parkins et al. [39] we investigated this mouse model using luciferase-positive 231BR cells and 323 measured a strong correlation on day 0 between the number of signal voids detected in day 0 brain MR images and 324 the brain signal measured in bioluminescence images, which is only detected from viable cells, providing evidence 325 that signal voids represent live iron-labeled cells. In Hamilton et al. [41] we used fluorescence activated cell sorting 326 (FACS) to successfully isolate live 231BR cells that were GFP-positive and labeled with red fluorescent (Flash Red) 327 MPIO from the brains of mice. The GFP+ Flash Red+ cells were collected and expanded in vitro. The majority 328 ( $90 \%)$ of cells adhered to tissue culture plastic and successfully expanded, displaying the same cell morphology as 329 the original cultured 231BR cells. This provide additional support for our claim that signal voids in MRI represent 330 viable iron-positive cancer cells.

331 Our previous work has shown that the number of signal voids detected in MRI of the brain decreases over the course 332 of the experiment, from day 0 to day 8 , which is expected as the large majority of cancer cells that arrest in the brain 333 do not survive and are cleared with time [39]. The signal voids which do persist in the brain over time are thought to 334 represent iron-retaining, non-proliferative or dormant cancer cells. These cells have been shown to contribute to 335 tumor recurrence [41]. It appears that both nude and NSG mice are capable of clearing dead cells from the brain.

337 3D MRI of the entire mouse brain at high resolution allowed us to view brain metastases in all 3 orientations and to digitally re-slice images to carefully interrogate image data. We counted and measured the volume of all MRI-

339 detectable brain metastases. Brain metastases developed more quickly in NSG mice. At day 21 post cell injection 340 NSG mice had more than 3 times the number of brain metastases. The NK cells and the remaining innate immune 341 cells in nude mice likely contribute to the reduced tumor growth at this timepoint. This finding is in agreement with 342 the results of other groups that show that more immune compromised mouse models are more permissive for tumor 343 growth and metastasis [42-45]. Puchalapalli et al. [45] have previously reported an increase in the metastatic burden 344 (in liver, lungs, brain and bones) in NSG compared to nude mice that were injected with the parental 231 breast 345 cancer cell line in an intracardiac experimental metastasis model. In this study, metastases in each organ were 346 enumerated from ex vivo fluorescence microscopy images. Our work demonstrates the advantages of using in vivo 347 and longitudinal 3D MRI for the accounting of metastases in the whole brain. 
349 NSG mice had to be euthanized at day 21/22. This was unexpected, as volumetric analyses of the tumor burden in

350 the brain at this timepoint compared to the tumor burden of the nude mice at their endpoint of day 32 indicated that

351 the NSG mice had lesser tumor burden in the brain. This suggested that there was a possibility of metastases to have

352 occurred elsewhere in the body that could be contributing to a greater disease burden. Mouse body MRI confirmed 353 tumors in the liver, lungs, and lymph nodes.

355 Nude mice were imaged at a third timepoint. On day 32 the number of brain metastases in nude mice was similar to 356 the number of brain metastases in NSG mice on day 21. The mean volume of the brain metastases in the nude mice 357 on day 32 was more than 3 times greater than those in NSG mice at day 21, having had more time to develop. 358 Overall, the brain tumor burden at necessary endpoint was significantly greater in nude mice.

360 Ex vivo images of livers removed on the day of the intracardiac injection of iron labeled 231BR cells revealed 361 numerous signal voids in all lobes of the liver in both strains of mice indicating that there was similar cancer cell arrest for both strains at this timepoint. We did not do a quantitative analysis of liver signal voids, however, in a

363 previous study where we injected MPIO-labeled melanoma cells via the mesenteric vein, mouse livers were imaged 364 ex vivo by MRI and the signal void area was shown to correlate with the number of cells injected [46]. Ex vivo 365 images of livers removed at endpoint showed that numerous metastases formed in NSG mice while no liver 366 metastases could be detected in nude mice.

367 The 231BR cell line was developed to selectively grow distant metastases in the brains of nude mice, and so the 368 proliferation of metastases of this cell line in the liver demonstrates the loss of selectivity to the brain in the NSG 369 mouse.

\section{$371 \quad$ Conclusion}

372 In summary, high resolution cellular MRI allowed us to characterize the 231BR cell line in both the NSG and nude 373 mouse models. We found marked differences in tumor incidence, volumes, and body tumor burden between strains.

374 Our in vivo comprehensive analysis of cancer cell arrest, clearance, and tumor progression is important for 375 understanding the metastatic cascade of a model of breast cancer brain metastasis that can be challenging to obtain 376 with in vitro or ex vivo methods alone. 


\section{$378 \quad$ List of Abbreviations}

379 Abbreviations

380 bSSFP

381 BW

382 DMEM

383 FA

384 FACS

385 FIESTA

386 GFP

$387 \quad \mathrm{H} \& \mathrm{E}$

388 MPIO

389 MRI

$390 \quad$ NSG

$391 \quad$ PBS

392 PDX

393 RF

394 TE

395 TR

396 US

397 231BR

398

\section{Full Form}

balanced steady-state free precession

bandwidth

Dulbecco's modified Eagle's medium

flip angle

fluorescence activated cell sorting

Fast Imaging Employing Steady State Acquisition

green fluorescent protein

hematoxylin and eosin

micron-sized iron oxide particles

magnetic resonance imaging

NOD/SCID/ILIIrg-/-

Perl's Prussian Blue

patient-derived xenograft

radiofrequency

echo time

repetition time

ultrasound

MDA-MB-231BR 


\section{Declarations}

$400 \quad$ Funding

401 This study was supported by the Canadian Institute for Health Research (P.J. Foster) and the Breast Cancer Society

402 of Canada (N.N. Knier).

403

\section{Conflicts of interest/Competing interests}

405 The authors declare that they have no competing interests.

\section{$407 \quad$ Ethics approval}

408 Animals were cared for in accordance with the standards of the Canadian Council on Animal

409 Care, and under an approved protocol of the Western University's Council on Animal Care (2018-135).

\section{Consent to participate}

412 Not applicable

\section{Consent for publication}

415 Not applicable

\section{Availability of data and materials}

418 The datasets used and/or analysed during the current study are available from the corresponding author on

419 reasonable request.

\section{Code availability}

422 Not applicable

\section{Author's Contributions}

425 P.J.F. designed the experiments. N.N.K, P.J.F, and A.M.H conducted the experiments. N.N.K. analyzed the data.

426 N.N.K. and P.J.F. wrote the main manuscript text. All authors reviewed the manuscript. 


\section{Acknowledgements}

428 The authors would like to thank Ashley V. Makela for assisting with mouse injections for this study and the

429 Canadian Institute for Health Research and the Breast Cancer Society of Canada for their funding. 
1. Patanaphan V, Salazar OM, Risco R. Breast cancer: metastatic patterns and their prognosis. Southern medical journal. 1988;81(9):1109-1112.

2. Gavrilovic IT, Posner JB. Brain metastases: epidemiology and pathophysiology. Journal of neurooncology. 2005;75(1):5-14.

3. Nayak L, Lee EQ, Wen PY. Epidemiology of brain metastases. Current oncology reports. 2012;14(1):4854.

4. Tabouret E, Chinot O, Metellus P, Tallet A, Viens P, Goncalves A. Recent trends in epidemiology of brain metastases: an overview. Anticancer research. 2012;32(11):4655-4662.

5. Anders C, Carey LA. Understanding and treating triple-negative breast cancer. Oncology (Williston Park, NY). 2008;22(11):1233.

6. Heitz F, Harter P, Lueck H-J, Fissler-Eckhoff A, Lorenz-Salehi F, Scheil-Bertram S, et al. Triple-negative and HER2-overexpressing breast cancers exhibit an elevated risk and an earlier occurrence of cerebral metastases. European journal of cancer. 2009;45(16):2792-2798.

7. Loibl S, Gianni L. HER2-positive breast cancer. The Lancet. 2017;389(10087):2415-2429.

8. Chambers AF, Groom AC, MacDonald IC. Dissemination and growth of cancer cells in metastatic sites. Nature Reviews Cancer. 2002;2(8):563-572.

9. Goss PE, Chambers AF. Does tumour dormancy offer a therapeutic target? Nature Reviews Cancer. 2010;10(12):871-877.

10. Fehm T, Mueller V, Marches R, Klein G, Gueckel B, Neubauer H, et al. Tumor cell dormancy: implications for the biology and treatment of breast cancer. Apmis. 2008;116(7-8):742-753.

11. Brackstone M, Townson JL, Chambers AF. Tumour dormancy in breast cancer: an update. Breast Cancer Research. 2007;9(3):208.

12. Lockman PR, Mittapalli RK, Taskar KS, Rudraraju V, Gril B, Bohn KA, et al. Heterogeneous blood-tumor barrier permeability determines drug efficacy in experimental brain metastases of breast cancer. Clinical cancer research. 2010;16(23):5664-5678.

13. Burdall SE, Hanby AM, Lansdown MR, Speirs V. Breast cancer cell lines: friend or foe? Breast cancer research. 2003;5(2):89.

14. Pelleitier M, Montplaisir S. The nude mouse: a model of deficient T-cell function. Methods and achievements in experimental pathology. 1975;7:149-166.

15. Yoneda T, Williams PJ, Hiraga T, Niewolna M, Nishimura R. A bone-seeking clone exhibits different biological properties from the MDA-MB-231 parental human breast cancer cells and a brain-seeking clone in vivo and in vitro. Journal of bone and mineral research. 2001;16(8):1486-1495.

16. Kumar S, Bajaj S, Bodla RB. Preclinical screening methods in cancer. Indian journal of pharmacology. 2016;48(5):481.

17. Gillet J-P, Varma S, Gottesman MM. The clinical relevance of cancer cell lines. Journal of the National Cancer Institute. 2013;105(7):452-458. 
18. Siolas D, Hannon GJ. Patient-derived tumor xenografts: transforming clinical samples into mouse models. Cancer research. 2013;73(17):5315-5319.

19. Sulaiman A, Wang L. Bridging the divide: preclinical research discrepancies between triple-negative breast cancer cell lines and patient tumors. Oncotarget. 2017;8(68):113269.

20. Weeber F, Ooft SN, Dijkstra KK, Voest EE. Tumor organoids as a pre-clinical cancer model for drug discovery. Cell chemical biology. 2017;24(9):1092-1100.

21. Ruggeri BA, Camp F, Miknyoczki S. Animal models of disease: pre-clinical animal models of cancer and their applications and utility in drug discovery. Biochemical pharmacology. 2014;87(1):150-161.

22. Sharpless NE, DePinho RA. The mighty mouse: genetically engineered mouse models in cancer drug development. Nature reviews Drug discovery. 2006;5(9):741-754.

23. Dobrolecki LE, Airhart SD, Alferez DG, Aparicio S, Behbod F, Bentires-Alj M, et al. Patient-derived xenograft (PDX) models in basic and translational breast cancer research. Cancer and Metastasis Reviews. 2016;35(4):547-573.

24. Whittle JR, Lewis MT, Lindeman GJ, Visvader JE. Patient-derived xenograft models of breast cancer and their predictive power. Breast cancer research. 2015;17(1):17.

25. Jung J, Seol HS, Chang S. The generation and application of patient-derived xenograft model for cancer research. Cancer research and treatment: official journal of Korean Cancer Association. 2018;50(1):1.

26. Yu J, Qin B, Moyer AM, Sinnwell JP, Thompson KJ, Copland JA, et al. Establishing and characterizing patient-derived xenografts using pre-chemotherapy percutaneous biopsy and post-chemotherapy surgical samples from a prospective neoadjuvant breast cancer study. Breast Cancer Research. 2017;19(1):130.

27. Contreras-Zárate MJ, Ormond DR, Gillen AE, Hanna C, Day NL, Serkova NJ, et al. Development of novel patient-derived xenografts from breast cancer brain metastases. Frontiers in oncology. 2017;7:252.

28. Hoffmann J, Fichtner I, Lemm M, Lienau P, Hess-Stumpp H, Rotgeri A, et al. Sagopilone crosses the blood-brain barrier in vivo to inhibit brain tumor growth and metastases. Neuro-oncology. 2009;11(2):158-166.

29. Naumov GN, MacDonald IC, Weinmeister PM, Kerkvliet N, Nadkarni KV, Wilson SM, et al. Persistence of solitary mammary carcinoma cells in a secondary site: a possible contributor to dormancy. Cancer research. 2002;62(7):2162-2168.

30. Shafie SM, Grantham FH. Role of hormones in the growth and regression of human breast cancer cells (MCF-7) transplanted into athymic nude mice. Journal of the National Cancer Institute. 1981;67(1):51-56.

31. Murrell DH, Foster PJ, Chambers AF. Brain metastases from breast cancer: lessons from experimental magnetic resonance imaging studies and clinical implications. Journal of molecular medicine. 2014;92(1):5-12.

32. Foster-Gareau P, Heyn C, Alejski A, Rutt BK. Imaging single mammalian cells with a $1.5 \mathrm{~T}$ clinical MRI scanner. Magnetic Resonance in Medicine: An Official Journal of the International Society for Magnetic Resonance in Medicine. 2003;49(5):968-971.

33. McFadden C, Mallett CL, Foster PJ. Labeling of multiple cell lines using a new iron oxide agent for cell tracking by MRI. Contrast media \& molecular imaging. 2011;6(6):514-522.

34. Makela AV, Murrell DH, Parkins KM, Kara J, Gaudet JM, Foster PJ. Cellular imaging with MRI. Topics in Magnetic Resonance Imaging. 2016;25(5):177-186. 
35. Heyn C, Ronald JA, Ramadan SS, Snir JA, Barry AM, MacKenzie LT, et al. In vivo MRI of cancer cell fate at the single-cell level in a mouse model of breast cancer metastasis to the brain. Magnetic Resonance in Medicine: An Official Journal of the International Society for Magnetic Resonance in Medicine. 2006;56(5):1001-1010.

36. Murrell DH, Zarghami N, Jensen MD, Dickson F, Chambers AF, Wong E, et al. MRI surveillance of cancer cell fate in a brain metastasis model after early radiotherapy. Magnetic resonance in medicine. 2017;78(4):1506-1512.

37. Economopoulos V, Chen Y, McFadden C, Foster PJ. MRI detection of nonproliferative tumor cells in lymph node metastases using iron oxide particles in a mouse model of breast cancer. Translational oncology. 2013;6(3):347.

38. Basse P, Hokland P, Heron I, Hokland M. Fate of tumor cells injected into left ventricle of heart in BALB/c mice: role of natural killer cells. JNCI: Journal of the National Cancer Institute. 1988;80(9):657-665.

39. Parkins KM, Hamilton AM, Makela AV, Chen Y, Foster PJ, Ronald JA. A multimodality imaging model to track viable breast cancer cells from single arrest to metastasis in the mouse brain. Scientific reports. 2016;6(1):1-9.

40. Murrell DH, Hamilton AM, Mallett CL, van Gorkum R, Chambers AF, Foster PJ. Understanding heterogeneity and permeability of brain metastases in murine models of HER2-positive breast cancer through magnetic resonance imaging: implications for detection and therapy. Translational oncology. 2015;8(3):176-184.

41. Hamilton AM, Parkins KM, Murrell DH, Ronald JA, Foster PJ. Investigating the impact of a primary tumor on metastasis and dormancy using MRI: new insights into the mechanism of concomitant tumor resistance. Tomography. 2016;2(2):79.

42. Simpson-Abelson MR, Sonnenberg GF, Takita H, Yokota SJ, Conway TF, Kelleher RJ, et al. Long-term engraftment and expansion of tumor-derived memory $\mathrm{T}$ cells following the implantation of non-disrupted pieces of human lung tumor into NOD-scid IL2R $\gamma$ null mice. The Journal of Immunology. 2008;180(10):7009-7018.

43. Agliano A, Martin-Padura I, Mancuso P, Marighetti P, Rabascio C, Pruneri G, et al. Human acute leukemia cells injected in NOD/LtSz-scid/IL-2R $\gamma$ null mice generate a faster and more efficient disease compared to other NOD/scid-related strains. International journal of cancer. 2008;123(9):2222-2227.

44. Quintana E, Shackleton M, Sabel MS, Fullen DR, Johnson TM, Morrison SJ. Efficient tumour formation by single human melanoma cells. Nature. 2008;456(7222):593-598.

45. Puchalapalli M, Zeng X, Mu L, Anderson A, Glickman LH, Zhang M, et al. NSG mice provide a better spontaneous model of breast cancer metastasis than athymic (nude) mice. PLoS One. 2016;11(9).

46. Townson JL, Ramadan SS, Simedrea C, Rutt BK, MacDonald IC, Foster PJ, Chambers AF. Threedimensional imaging and quantification of both solitary cells and metastases in whole mouse liver by magnetic resonance imaging. Cancer research. 2009 Nov 1;69(21):8326-31. 


\section{Figure 1}

A
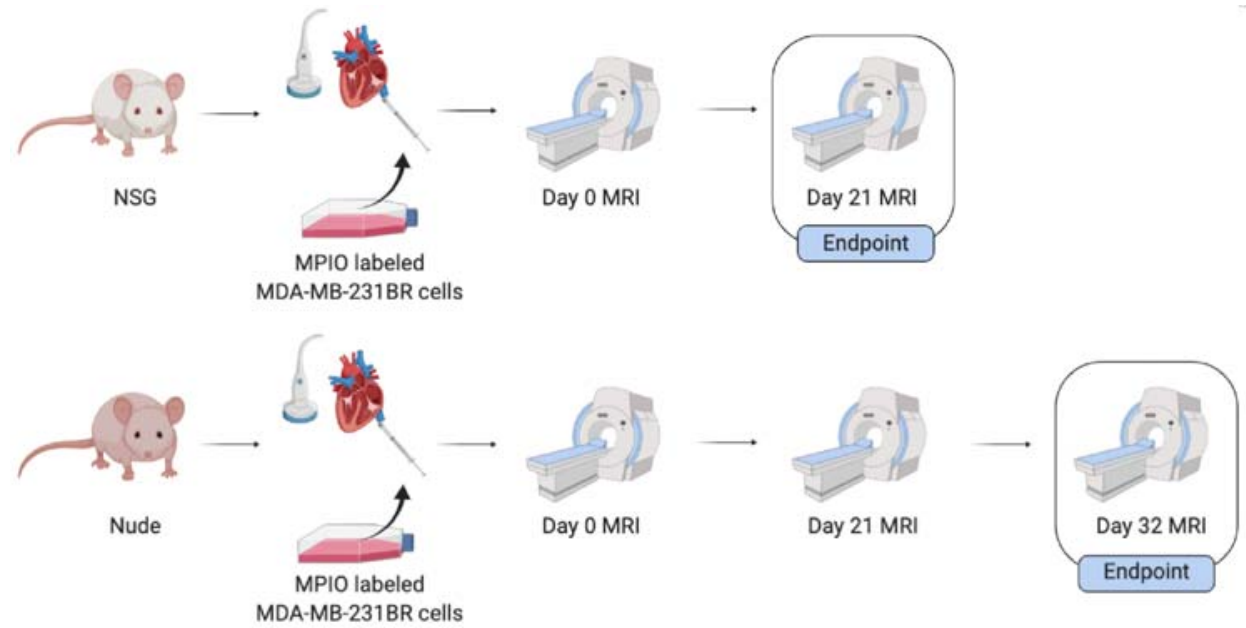

Figure. 1 Experimental design for the NSG and nude mouse model 


\section{Figure 2}

A

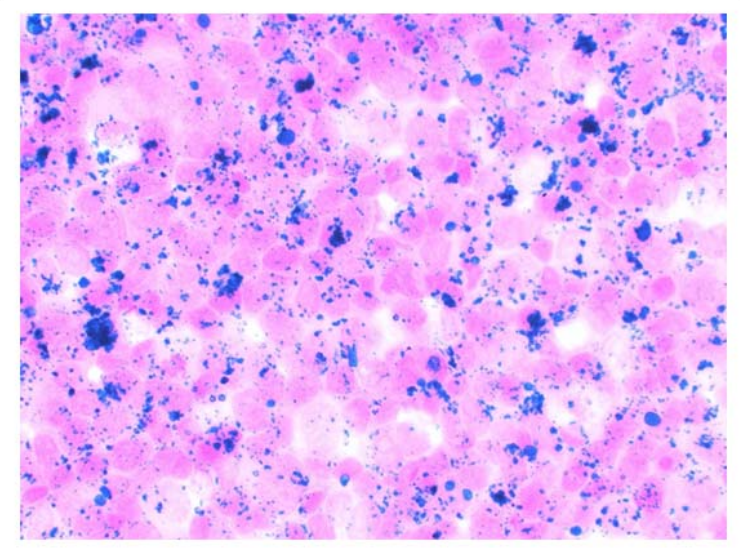

B

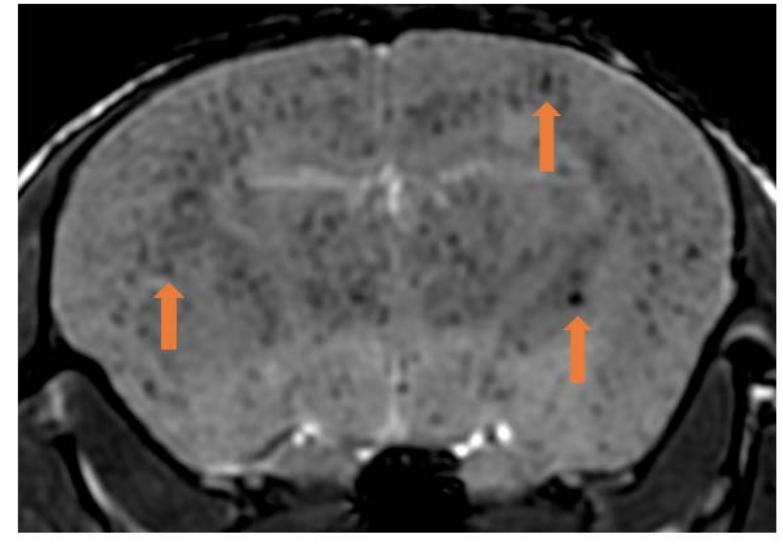

Figure. 2 Iron labeling and injection of 231BR cells (A) Perl's Prussian Blue stain showing intracellular iron (blue, PPB) detected within MDA-MB-231BR cells (pink, Nuclear fast red) in vitro. (B) Representative day 0 image showing visualization of successful iron-labeled cancer cell delivery by intracardiac injection as regions of signal void (orange arrows) 
Figure 3

A Day 0

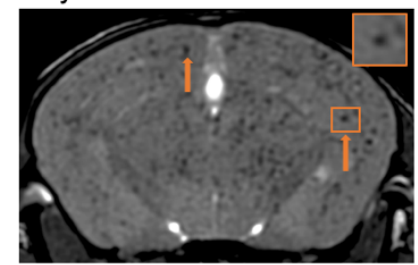

Day 0

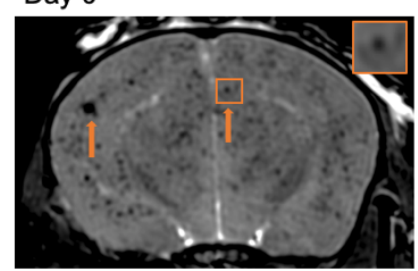

Endpoint (Day 21)

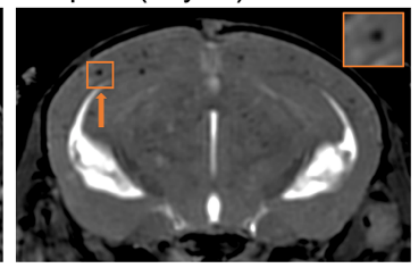

Endpoint (Day 32)

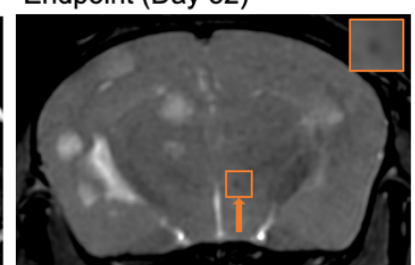

B

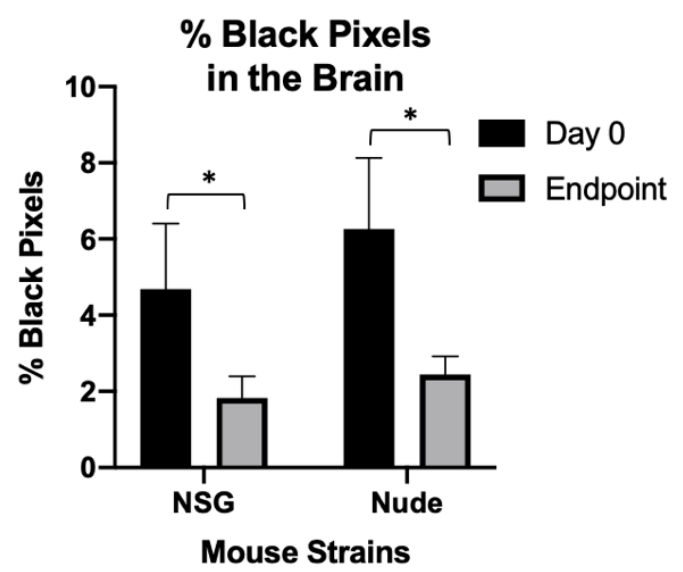

Figure. 3 Cancer cell arrest and clearance (A) Balanced steady-state free precession images showing initial

599 cancer cell arrest at day 0 and clearance and retention at endpoint. The orange arrows indicate regions of signal void.

600 (B) The percentage of black pixels, representing regions of signal void was not significantly different between

601 strains, however there was a significant decrease within strains from day 0 to endpoint, suggesting similar cancer

602 cell arrest and clearance between nude and NOD/SCID/ILIIrg-/- mice 
Figure 4

A

\section{Day 21}

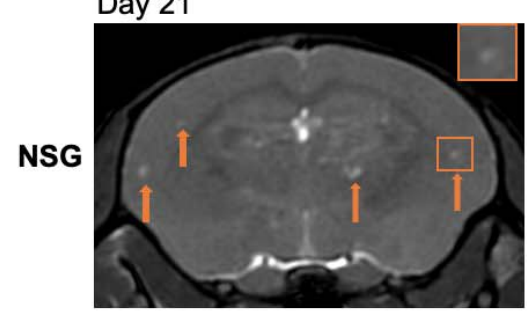

\section{Day 21}

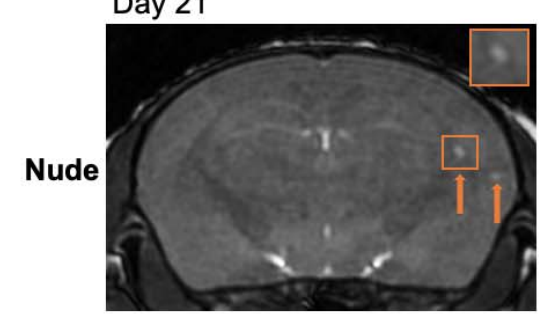

B

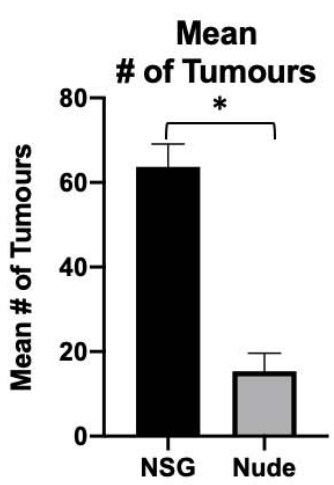

C

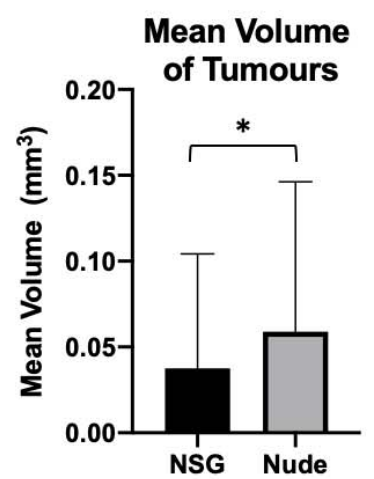

D

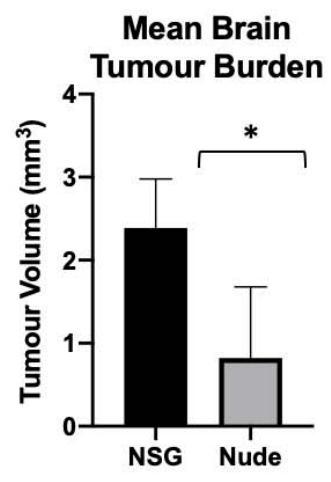

603

604

605

606

607

608

609

Figure. 4 Day 21 visualization and quantification of brain metastases (A) Balanced steady-state free precession images showing brain metastases in both strains of mice as regions of signal hyperintensity (orange arrows) at day 21. (B) There were significantly more brain metastases on average detected with MRI in NSG mice than nude mice at day 21. (C) The mean volume of tumors present in the brain was significantly greater in nude mice than NSGs.

(D) The brain tumor burden of NOD/SCID/ILIIrg-/- mice was significantly higher than in nude mice at day 21. Data is presented as mean + - SEM. *indicates $\mathrm{p}<0.05$ 
Figure 5

A

\section{Day 21}

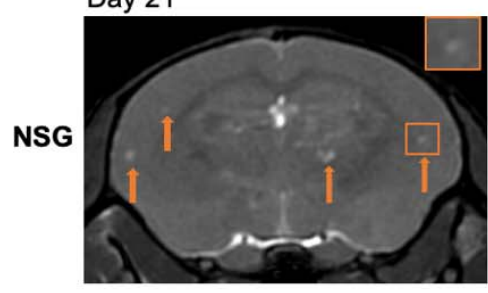

\section{Day 32}

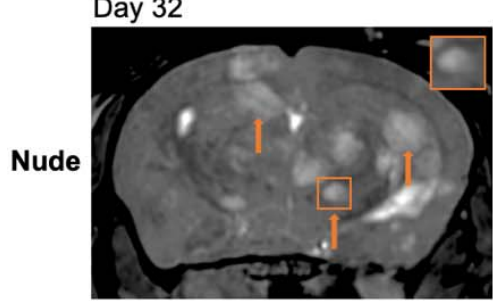

B

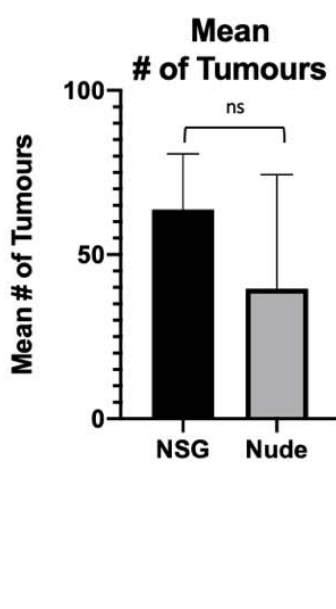

C

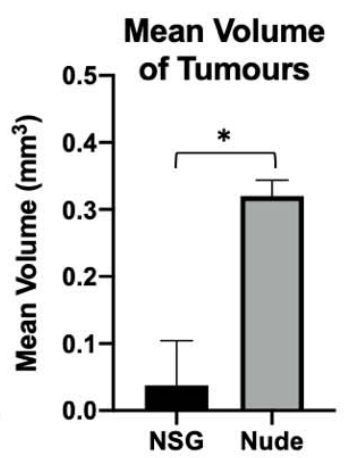

D

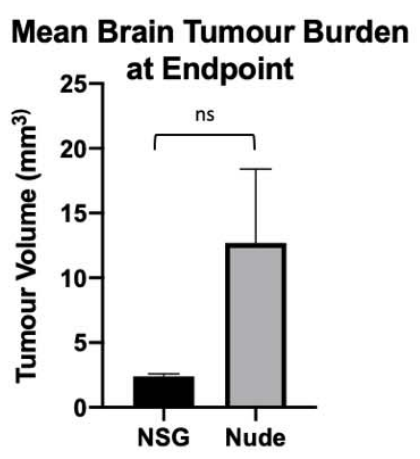

Figure. 5 Endpoint visualization and quantification of brain metastases (A) Magnetic resonance images of both

612 NOD/SCID/ILIIrg-/- and nude mice at each strain's respective endpoints (Day 21 for NOD/SCID/ILIIrg-/-, Day 32

613 for nude) showing regions of signal hyperintensity where brain metastases have developed (orange arrows). (B)

614 There was no significant difference between the number of brain metastases detected with magnetic resonance

615 imaging between strains at endpoint. (C) The tumors in the nude mice were significantly larger in volume than those

616 in the NOD/SCID/ILIIrg-/- mice. (D) The total tumor burden within the brain at endpoint in both nude and mice was

617 not significantly different. Data is presented as mean + - SEM. *indicates $\mathrm{p}<0.05$ 


\section{Figure 6}
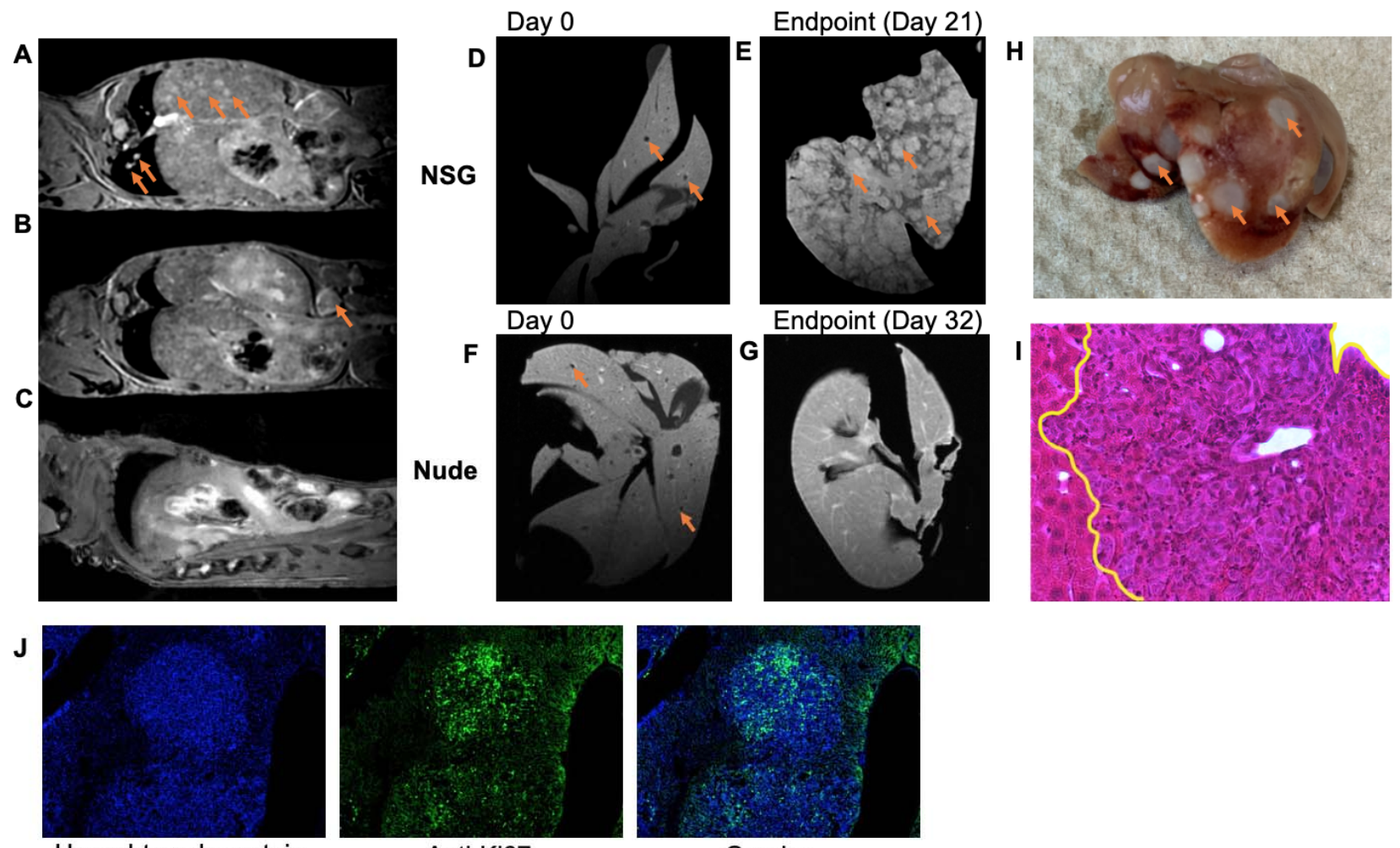

Anti-Ki67

Overlay

Figure. 6 Body and liver imaging with correlative histology and immunofluorescence (A) Body imaging of

NOD/SCID/ILIIrg-/- mouse allowed for detection of significant tumor burden in the liver, lungs. (B) Tumors were detected in the lymph nodes of NOD/SCID/ILIIrg-/- mice with magnetic resonance imaging. (C) No tumors were

622 detected with magnetic resonance imaging in the nude mouse body. (D) Spoiled gradient echo sequence image

623 showing initial arrest of cancer cells in ex vivo liver at Day 0 for NOD/SCID/ILIIrg-/- mouse (E) Spoiled gradient

624 echo sequence image of ex vivo liver showing regions of hyperintensity where liver metastases have developed in

625 the NOD/SCID/ILIIrg-/- mouse. (F) Day 0 spoiled gradient echo sequence image of ex vivo liver of from a nude

626 mouse showing signal voids, representing arrested cancer cells. (G) Spoiled gradient echo sequence image of Day

62732 (endpoint) ex vivo liver of a nude mouse with no magnetic resonance detectable metastases $(\mathrm{H})$ Photo of a

628 representative ex vivo liver from an NOD/SCID/ILIIrg-/- mouse showing tumors on the exterior of the organ. (I)

629 H\&E-stained section showing liver metastases that had developed in the NOD/SCID/ILIIrg-/- mouse (outlined in

630 yellow). (J) Ki67 staining showing a highly proliferative tumor within the NOD/SCID/ILIIrg-/- liver 